\title{
On the brightness of short and long flashes'
}

JEREMY M. ANGLIN AND R. J. W. MANSFIELD 2,3 HAR VARD UNIVERSITY

The equation proposed by $S$. S. Stevens to describe the relations among brightness, intensity, and duration involves assumptions that are here made explicit. The equation is shown to apply to earlier studies designed to measure critical duration.

Bloch's law, the Broca Sulzer effect, and a decrease in critical duration with increasing luminance have been demonstrated for supraliminal stimulus values (Aiba \& S. S. Stevens, 1964; Raab, 1962; J. C. Stevens \& Hall, 1966). Although the study by Aiba and Stevens used interocular brightness matching, whereas the other two studies used magnitude estimation, similar results were obtained. The results of all three experiments accord with a simple model, schematized in Fig. 1, which is designed to represent the relation among brightness, intensity, and duration ( $\mathbf{S}$. S. Stevens, $1966 \mathrm{a}$, b). The purpose of this note is to examine the equation that appeared in conjunction with that model and to discuss the model in the light of experimental evidence.

In Fig. 1, the positive slope $(\tan a)$ and the parallelism of the lines in the region of short duration result from the dependence of brightness upon the product of luminance and duration (Bloch's law). Along each line the peak luminance of the flash is assumed to be constant. For stimuli of long duration the lines become horizontal, and the parameter reffects the psychophysical power law relating brightness, $B$, to luminance, $L$ :

$$
B=k\left(L-L_{0}\right) \cdot 33
$$

where $L_{0}$ is the effective threshold, and $k$ is a constant that depends upon the units of $B$ and $L$. The resulting two families of parallel lines meet at the critical duration, represented by the slanted dashed line. The size of the angle $\theta$ thus becomes a measure of the dependence of the critical duration on the intensity of the flash. The angle 8 has an interesting effect on the psychophysical power law, for a different exponent is required at short durations from that required at long durations. These relations may be seen as follows.

In Fig. 1 the length of the heavy line $F$ is proportional to the flash exponent, the line $S$ is proportional to the steady-state exponent, $\tan a$ is equal to the slope of the family of parallel lines in the Bloch region, and $\tan \theta$ is equal to the absolute value of the slope of the straight line drawn through the critical durations. From the diagram, it can be seen that:

and therefore

$$
\tan a=\mathrm{BC} / \mathrm{BD}=(\mathrm{F}-\mathrm{S}) / \mathrm{BD}
$$

Furthermore

$$
\mathrm{BD}=\mathrm{AE}=(\mathrm{F}-\mathrm{S}) / \tan a
$$

Hence

$$
\tan \theta=\mathrm{AE} / \mathrm{ED}=(\mathrm{F}-\mathrm{S}) / \mathrm{S} \tan a
$$

$$
\mathrm{F} / \mathrm{S}=1+\tan a \tan \theta
$$

Equation (2) shows that the relation of the exponent for short flashes in the Bloch region to the exponent for long flashes is determined by the size of the angle $\theta$.

Below the critical duration, luminance can be traded for duration (Bloch's law). Therefore, the parallel lines in the Bloch region, which are power functions relating brightness to duration for flashes of constant peak luminance, must have, the same siope as the functions relating brightness to luminance for constant duration. That is to say, the straight parallel lines in the short-duration region of Fig. 1 indicate that brightness grows as a power function of duration with an exponent equal to $\tan a$. In this region it follows from Bloch's law that, for constant duration, brightness grows as a power function of intensity with the same exponent $(\tan a)$.

At this point it should be noted that the distances $F$ and $S$ in Fig. 1, which are proportional to the flash and steady-state exponents, have been conveniently set equal to them. Thus

$$
\mathrm{F}=\tan a
$$

This step was not made explicit in earlier discussions and its omission has led to occasional confusion. By substituting $F$ for tan $a$ in Equation (2) we obtain the formula that Stevens published:

$$
1 / S=1 / F+\tan \theta
$$

Stevens' model can also be used to determine the functional relation between the critical duration, $t_{c}$, and luminance, $L$. If we neglect the Broca Sulzer effect and match the expression for brightness in the Bloch region to the expression for brightness with the same luminance in the steady-state region, we have

$$
\mathrm{k}\left(\mathrm{L} . \mathrm{t}_{c}\right)^{\mathrm{F}}=\mathbf{k}^{\prime} \mathrm{L}^{\mathrm{S}}
$$

where $k$ and $k^{\prime}$ are constants that depend on the units of luminance, duration, and brightness. Further we may write

$$
\mathrm{t}_{\mathrm{c}}=\mathrm{k}^{\prime \prime} \mathrm{L}(\mathrm{S} / \mathrm{F}-\mathrm{1})
$$

where $k^{\prime \prime}$ is a constant that depends on the units of $L$ and $t_{c}$. That is to say, the critical duration changes as a power function of luminance with an exponent equal to $S / F-1$.

\section{Comparison with Other Studies}

A number of studies have dealt with the relation between critical duration and luminance. Graham and Kemp (1938), Keller (1941), and Herrick (1956) have each examined differential brightness thresholds for short flashes of varying duration as a function of adapting luminance. As shown in Fig. 2, all three studies found that critical duration decreased with adapting luminance.

The experiments by Graham and Kemp and by Keller were essentially similar. The observer viewed two separately illuminated semicircles that fell within the limits of the fovea (target diameter about $1.5 \mathrm{deg}$ ). The task was to detect a flash increment that was superimposed on one of the semicircles. The threshold flash intensity was determined for a number of durations and prevailing intensities of the two semicircles. Observations were binocular in Graham and Kemp's study, monocular in Keller's. The results of both experiments are shown in Fig. 2 . Keller noted that critical duration changes as a power function of luminance with an exponent of -0.12 . In the $\log -\log$ coordinates of Fig. 2 the data of

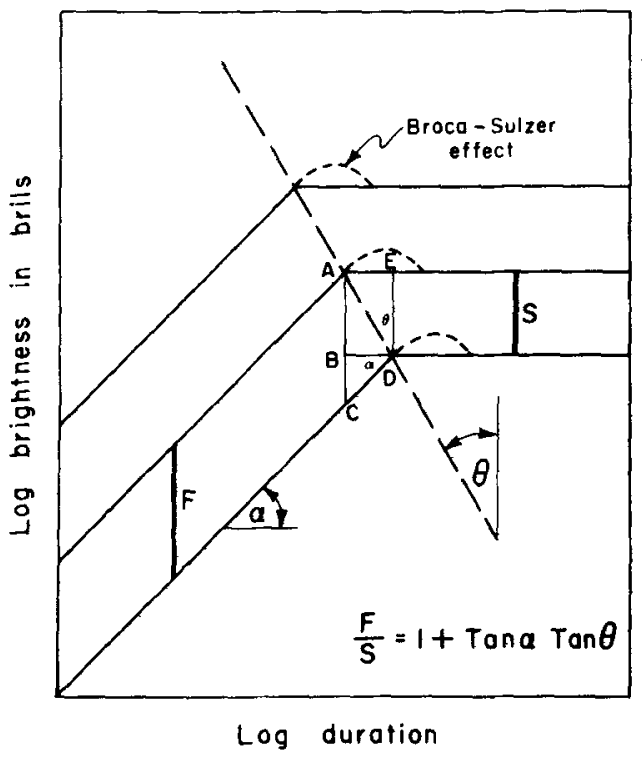

Fig. 1. Theoretical relation between the exponent governing the brightness of short flashes and the exponent for long steady-state flashes. In these $\log -\log$ coordinates the distances $F$ and $S$ are proportional to the respective exponents. As the angle $\theta$ increases, the vertical distance $F$ increases according to Equation (2). 


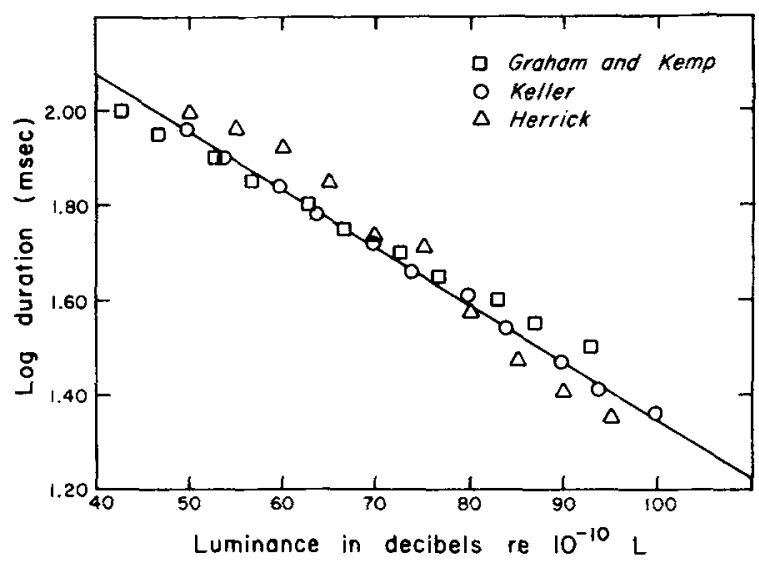

Fig. 2. Critical duration as a function of luminance for three studies that used the method of differential brightness thresholds. The values for Graham and Kemp and for Keller have been taken directly from the original papers. The values for Herrick represent the geometric means of his two observers. In these log-log coordinates the solid straight line represents a power function.

Graham and Kemp also follow a power function, in that the points can be fitted quite well by a straight line with a slope of -0.10 .

Herrick used a different arrangement. He superimposed flash increments or decrements on a steadily illuminated 1 -deg field and determined the differential threshold for various durations and for 10 levels of adapting illumination. The geometric means of the estimated critical durations for his two observers are shown as triangles in Fig. 2. The data approximate a power function with an exponent of about 0.15 .

The three slopes (exponents) exhibited by the data in Fig. 2 can be used to derive values for flash exponents on the assumption that the steady-state exponent is 0.33 . From Equation (6) the flash exponent, F, equals the steady-state exponent, S, divided by 1 plus the critical duration exponent. The calculated flash exponents are $0.38,0.37$, and 0.39 .

These three studies support Stevens' model in that they suggest (1) that the critical duration changes as a power function of luminance and (2) that the flash exponent is somewhat larger than the steady-state exponent. On the other hand, the values of the flash exponents suggested by these experiments are uniformly lower than those obtained in the matching experiments cited above. The experiment of Aiba and Stevens gave a flash exponent of 0.4, that of Raab, 0.5, and that of J. C. Stevens and Hall, 0.45. It should be noted, of course, that neither in the matching nor in the threshold experiments were the exponents determined with great precision.

The apparent systematic discrepancy between the two sets of flash exponents may well be the result of the different procedures employed in the two types of studies. One set of experiments concerned the differential threshold, whereas the other involved the supraliminal matching of brightness, either to numbers in the magnitude-matching studies, or to other flashes in the interocular brightness-matching study. In the threshold experiments the observer was light-adapted to the prevailing intensity of the background stimulus upon which the flashes were superimposed. In the matching experiments, on the other hand, the observer was essentially dark-adapted throughout the experiment. Changes in the state of adaptation have been shown to affect the exponent of the brightness function (J. C. Stevens \& Stevens, 1963) and to affect the critical duration (Aiba \& Stevens, 1966).

If the discrepancy between the values of the flash exponents obtained in the two types of experiments reflects nothing more than the parametric variations in the procedures employed, then the agreement concerning the general form of the relation between critical duration and luminance illustrates how Stevens' model may help in the integration of data on the critical duration obtained by very different procedures. On the other hand, a key assumption of the model, namely, that in the Bloch region brightness grows as a power function of duration for fixed luminance, has recently been questioned. Ekman (1960) has argued that the dependence of brightness upon time, in the short-duration region, is actually a logarithmic relation of the form:

$$
B=a+b \log t
$$

where brightness, $B$, is estimated by direct scaling methods.

Ekman reached his conclusion by analyzing the data from the study of Raab who had obtained magnitude estimations from observers for flashes of different durations. Ekman averaged the six (out of nine) highest median values of $\log$ brightness obtained by Raab and showed the resulting functions to be consistent with the logarithmic relation. In addition, he estimated the temporal threshold, $t_{0}$, for each of Raab's six highest peak luminances, using the relation $\log t_{0}=-a / b$ obtained from Equation (7). He found that the extrapolated thresholds for the three highest peak luminance levels appeared to satisfy Bloch's law. However, as Baumgardt (1967) points out, there is a "numerical error of the order of eighty in Ekman's extrapolated temporal thresholds," as compared with experimentally obtained values. Furthermore, the extrapolated temporal thresholds of the three lowest luminance levels appear to be inconsistent with Bloch's law. In fact, they indicate that brightness was roughly independent of flash energy. Yet the fitted logarithmic functions were taken as evidence that "the logarithmic relation . . . is verified for brightness perception" (Ekman, 1966).

A different treatment of Raab's data has led to a different conclusion (S. S. Stevens, 1966a). Raab's original raw data were used to calculate geometric means which are more efficient statistics than the medians used by Ekman. The geometric means gave a more linear relation between log brightness and log stimulus duration. Moreover, J. C. Stevens and Hall (1966) essentially replicated Rab's experiment and demonstrated, with geometric means, a linear relation between log brightness and log stimulus duration. Thus the two magnitude-estimation studies both suggest that, in the Bloch region, brightness grows as a power function of both duration and luminance. The exponent in the Bloch region has a value that definitely exceeds the value of the exponent in the steady-state region.

\section{REFERENCES}

AIBA, T. S., \& STEVENS, S. S. Relation of brightness to duration under light-'and dark-adaptation. Vision Res, 1964, 4, 391-401.

BAUMGARDT, E. On direct scaling methods. Vision Res, 1967, 7, 679-680.

EKMAN, G. Temporal integration of brightness. Vision Res, 1966, 6, $683-688$.

GRAHAM, C. H., \& KEMP, E. H. Brightness discrimination as a function of the duration of the increment in intensity. J. gen. Physiol., 1938, 21, 635-650.

HERRICK, R. M. Foveal luminance discrimination as a function of the duration of the decrement or increment in luminance. J. comp. physiol Psychol, 1956, 49, 437-443.

KELLER, M. The relation between the critical duration and intensity in brightness discrimination. J. exp. Psychol, 1941, 28, 407-418.

RAAB, D. Magnitude estimation of the brightness of brief foveal stimuli. Science, 1962, 135, 42-43.

STEVENS, J. C., \& HALL, J. Brightness and loudness as functions of stimulus duration. Percept. \& Psychophys, 1966, 1, 319-327.

STEVENS, J. C., \& STEVENS, S. S. Brightness function: Effects of -adaptation. J. Opt. Soc. Amer., 1963, 53, 375-385.

STEVENS, S. S. Duration, luminance and the brightness exponent. Percept. \& Psychophys., 1966a, 1, 96-100.

STEVENS, S. S. Concerning the measurement of brightness. J. Opt. Soc. Amer., 1966b, 56, 1135-1136.

\section{NOTES}

1. Both authors held National Research Council (Canada) Scholarships and wish to express their appreciation for this support.

2. This study was carried out in the Laboratory of Psychophysics with support from NSF Grant GB-3211 and NIH Grant NB-02974 (Laboratory of Psychophysics Report PPR-342).

3. Address: William James Hall, 33 Kirkland St., Harvard University, Cambridge, Mass. 02138.

(Accepted for publication June 10, 1968.) 